\title{
Experimental Characterization and Modeling of Outdoor-to-Indoor and Indoor-to-Indoor Distributed Channels
}

\author{
Claude Oestges, Nicolai Czink, Bernd Bandemer, Paolo Castiglione, \\ Florian Kaltenberger, and Arogyaswami J. Paulraj
}

\begin{abstract}
We propose and parameterize an empirical model of the outdoor-to-indoor and indoor-to-indoor distributed (cooperative) radio channel, using experimental data in the $2.4 \mathrm{GHz}$ band. In addition to the well-known physical effects of path loss, shadowing, and fading, we include several new aspects in our model that are specific to multi-user distributed channels: (i) correlated shadowing between different point to point links which has a strong impact on cooperative system performance, (ii) different types of indoor node mobility with respect to the transmitter and/or receiver nodes, implying a distinction between static and dynamic shadowing motivated by the measurement data, and (iii) a small-scale fading distribution that captures more severe fading than given by the Rayleigh distribution.
\end{abstract}

\section{INTRODUCTION}

Cooperative communication is a promising technology to increase coverage, reliability and spectral efficiency in next generation wireless networks [1], [2]. The basic idea is to allow nodes to "help" other nodes with their communication by exploiting the broadcast nature of the wireless channel. This approach can for example be used to establish collaboratively a reliable wireless link between a set of indoor nodes to a base station not necessarily in reach of the individual nodes [3],

This work was partially supported by the European Commission in the framework of the FP7 Network of Excellence in Wireless COMmunications NEWCOM++ (contract no. 216715), by US Army grant W911NF-07-2-00271, by the Vienna Science and Technology Fund in the FTW project PUCCO, and by the European COST 2100 Action. The FTW Forschungszentrum Telekommunikation Wien is supported by the Austrian Government and the City of Vienna within the competence center program COMET. The work of C. Oestges is supported by the Belgian Fonds de la Recherche Scientifique (FRS-FNRS). The work of N. Czink is supported by an Erwin Schroedinger Fellowship of the Austrian Science Fund (FWF grant number J2789-N14). The work of B. Bandemer is supported by an Eric and Illeana Benhamou Stanford Graduate Fellowship. The work of P. Castiglione is supported by the Austria Science Fund (FWF) through grant NFN SISE (S106). The work of F. Kaltenberger is supported by the European Commission in the framework of the FP7 project SENDORA (contract no. 216076) and by Eurecom.

Copyright (c) 2010 IEEE. Personal use of this material is permitted. However, permission to use this material for any other purposes must be obtained from the IEEE by sending a request to pubs-permissions @ ieee.org.

C. Oestges is with ICTEAM Electrical Engineering, Université catholique de Louvain (UCL), Louvain-la-Neuve, Belgium (email: claude.oestges@uclouvain.be).

N. Czink was with the Smart Antennas Research Group, Stanford University, Stanford, CA, USA. He is now with FTW Forschungszentrum Telekommunikation Wien, Vienna, Austria.

B. Bandemer and A.J. Paulraj are with the Smart Antennas Research Group, Stanford University, Stanford, CA, USA.

P. Castiglione is with FTW Forschungszentrum Telekommunikation Wien, Vienna, Austria.

F. Kaltenberger is with the Mobile Communications Department, Eurecom, Sophia-Antipolis, France.
[4], e.g. some nodes can be used as relays to the base station. In such networks, especially when the distributed multi-link channel is non-homogeneous, the selection of the best relay can even provide better performance compared to existing distributed space-time codes [5].

Yet, before developing algorithms tackling this challenge, the outdoor-to-indoor radio channel, as well as the channel between the cooperating distributed nodes (i.e. the distributed channel) must be measured and modeled. Most of the theoretical work on cooperative communications assumes lognormal shadowing and Rayleigh fading, where path-loss, shadowing and fading are all independent from one another. While this is often true in cellular mobile scenarios, where the Rayleigh assumption is used as a conservative model, this might not be the case in indoor cooperative channels, depending on the users' mobility. In this paper, we make a distinction between three types of mobility. In the first case, we consider a link between a mobile and a fixed terminal, i.e. either the receiver (Rx) or transmitter (Tx) is moving (this case is denoted as single-mobile), and it is expected that fading be mostly Rayleigh distributed, especially in non line-of-sight conditions. Yet, Rayleigh fading might not be prevalent in the two other types of mobility. The second type of mobility deals with moving terminals at both link ends (double-mobile links), and a preliminary analysis of the data showed that fading in such scenarios may sometimes be worse than Rayleigh. The last mobility is the nomadic case, where both terminals are static, although they can be located almost anywhere in the region of interest, most often in non line-of-sight from each other. In such channels, also known as fixed, fading is hardly characterized by Rayleigh statistics, even in non line-of-sight (NLOS) conditions. Indeed, Rx and Tx are static during any typical communication, as are most scatterers. So, temporal fading is only caused by the motion of some scatterers resulting in a Ricean fading distribution. A further difference to mobile links is that in mobile propagation, time and space are linked through the user's speed. By contrast, in nomadic systems, one must account for both the temporal fading and the nomadic aspect, i.e. the fact that the terminal can be used at many fixed positions, which results in spatial fading adding to the static path loss. Furthermore, it is important for nomadic channel models to account for possible cross-correlations between path-loss, shadowing, and fading statistics, as measurements reveal that such relationships exist.

A further particularity of cooperative systems is that, while 
the separation between nodes is large enough to decorrelate small-scale fading processes, shadowing correlation may be present and can significantly affect the performance of the network as shown in [6], [7].

Therefore, the goal of the present work is to analyze outdoor-to-indoor and indoor-to-indoor distributed measured channels and to infer a global empirical channel model, considering under a similar formalism both nomadic, single- and double-mobile indoor links. Our objective is also to derive a model able to fit as closely as possible the observed behaviors of the distributed channels, in terms of e.g. mobility type, timevarying vs. space-varying aspects, and shadowing correlation, so that the model can be used for system design. In [8], a model extrapolated from the present work has motivated the research of an appropriate grouping algorithm for cooperative indoor-to-outdoor networks. Simultaneously, our approach integrates existing site-specific models, so that despite its empirical nature, our model could be easily extended to different environments.

Related Work. Several papers have analyzed various properties of outdoor-to-indoor and indoor-to-indoor distributed channels. In [9], [10], the outdoor-to-outdoor channel was measured for static receivers, and models of the Ricean Kfactor were proposed. In [11], the long-term statistics of the fixed indoor channel were investigated. Mobile multilink measurements were presented in [12], for indoor MIMO channels with two base stations and two users, and in [13] for outdoor channels. In [14], various properties of indoor distributed (or peer-to-peer) channels have been analyzed for static nodes only: fading was modeled by a generalized gamma distribution, which might not be easily tractable.

Shadowing correlation has been studied extensively for nondistributed outdoor [15]-[18] and indoor [19] scenarios. A model for both outdoor and indoor fixed wireless channels is proposed in [6]. None of these studies take into account the impact of node mobility. The authors in [6] model the shadowing between any two nodes as a weighted line integral of a spatial loss field, which in turn is modeled as a wide sense stationary Gaussian random field. The formulation allows to calculate the shadowing correlation between any two link pairs. However, the model always produces a positive shadowing correlation, whereas our measurements also reveal negative correlations, and it has been shown in [7] that some cooperative protocols are extremely sensitive to the sign of the shadowing correlation.

Contributions. The analysis presented in this paper is the first one that includes both nomadic and mobile scenarios of outdoor-to-indoor as well as indoor-to-indoor distributed channels. The key contributions of the present paper are as follows.

- We investigate narrowband outdoor-to-indoor (O2I) and indoor-to-indoor (I2I) distributed channels based on a wideband experimental campaign at $2.4 \mathrm{GHz}$. Unlike previous models, we propose a unified framework that includes both nomadic and mobile links. The differences between the three types of mobility are also clearly highlighted in the modeling approach.

- While developing a detailed statistical model of the channel, we propose to separate static shadowing from dynamic shadowing, as suggested by the data analysis. The characterization of static shadowing and fading for nomadic channels also takes into account that space and time variations are caused by different mechanisms. This decomposition also permits a better characterization of dynamic shadowing correlation than previously published.

- For mobile I2I channels, we find that the experimental fading distribution follows the second-order scattering fading model presented in [20]. Furthermore, we show that the resulting model parametrization can be related to the number of moving nodes in the considered link, allowing a direct physical interpretation of the model.

- Despite the fact that complex distributions are used by the various models, they can always be expressed by means of normal variable generators, which makes them very simple to use.

Outline. Section II summarizes the experimental set-up. Section III details the general concept we use for the measurement analysis, while Section IV details the data postprocessing and the estimation of propagation metrics. Sections $\mathrm{V}-\mathrm{A}$ and $\mathrm{V}-\mathrm{B}$ present the extracted empirical models, respectively for the outdoor-to-indoor and the indoor distributed channels. Finally, Section VII summarizes this paper and draws conclusions.

\section{EXPERIMENTAL SET-UP}

This paper is based on channel measurements of the Stanford July 2008 Radio Channel Measurement Campaign. More details on the full campaign can be found in [21]. In this section, we briefly summarize the most important characteristics of the measurement set-up.

\section{A. Environments}

We measured two kinds of environments: O2I, downlink from a base station to distributed nodes, and I2I, between the distributed nodes.

1) Outdoor-to-Indoor: A representation of the outdoor area is given in Fig. 1. At the outdoor location, an array of two dualpolarized WiMAX base station antennas were mounted on a scissor lift raised to a height of $10 \mathrm{~m}$ (location 'Tx1' on Fig. 1), the arrow indicating the boresight orientation. The antenna 3 - $\mathrm{dB}$ beamwidth was 90 degrees in azimuth and 8 degrees in elevation. The gain in the direction of the main lobe was 15.5 $\mathrm{dBi}$, and the antenna was tilted to ensure that the indoor office was in the elevation direction of the main lobe.

The indoor environment was a typical cubicle-style office room (see Fig. 2), where the indoor terminals were distributed over the various cubicles and along one wall. The room size was $34 \mathrm{~m}$ by $15.7 \mathrm{~m}$; the ceiling height was $3 \mathrm{~m}$, while the height of the internal partition walls forming the cubicles, represented by light lines on the map, was $1.7 \mathrm{~m}$. Cubicle partition walls were constructed from metal frames and fabric-covered walls. Regarding the indoor receivers, the $8 \mathrm{Rx}$ ports of the sounder were used in two successive measurements, covering a total of 12 receive locations as represented in Fig. 2, with 


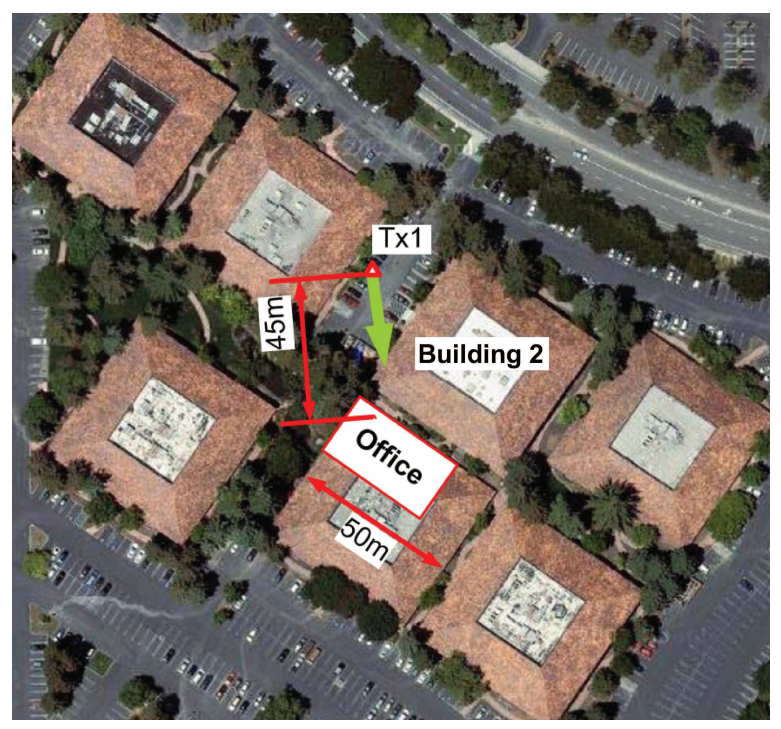

Fig. 1. Experimental outdoor environment

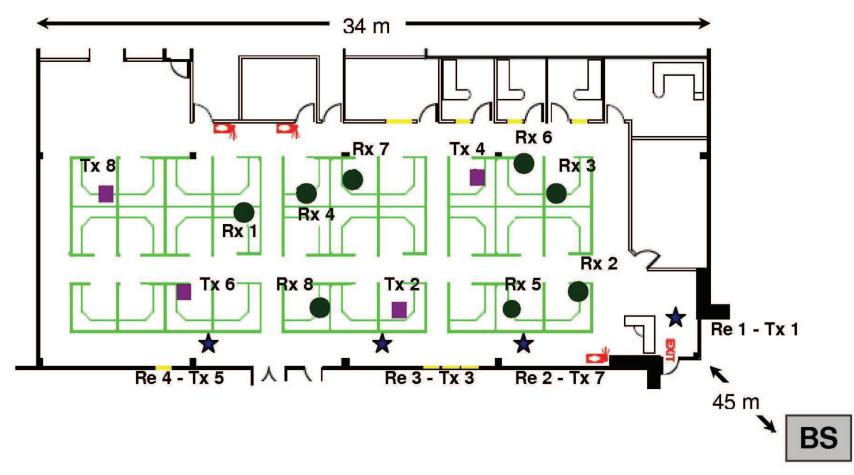

Fig. 2. Experimental set-up

circles and stars. The four receivers that are represented by stars were located at the wall close to the outdoor transmitter, and were kept at that position for the later I2I measurements. To avoid any confusion, they will be referred to as "relays" (with index 1 to 4). The indoor terminals (receivers and relays) used two different kinds of off-the-shelf vertically polarized omnidirectional WiFi antennas matched at $2.45 \mathrm{GHz}$. Their gain is $7 \mathrm{dBi}$ and $10 \mathrm{dBi}$, respectively, specified in the range of 2.4-2.83 GHz. To measure the distributed radio channel jointly, we connected the antennas to the switches using low-loss RF cables. During the measurements, the indoor terminals were kept static, while time variations were generated by people walking at a speed of $\approx 0.3 \mathrm{~m} / \mathrm{s}$ and carrying wooden boxes with aluminum frames. These were similar to briefcases in size, thus emulating people carrying their computer with them.

2) Indoor-to-Indoor: For the I2I segment, the measurements used the same WiFi antennas as those described above, and the same 8 (non-relay) receive locations as the O2I setup (represented by the circles in Fig. 2). The 8 Tx locations in the I2I set-up are represented by the stars (this time, the relays acted as transmitters), and the squares in Fig. 2. All nodes are in NLOS from each other. Three types of mobility are investigated:

- static measurements, where shadowing was generated by walking people carrying metal-framed objects (same as for O2I measurements),

- time-variant single-mobile measurements, where either all 8 receive or all 8 transmit antennas were moved randomly within a $2 \mathrm{~m}$ radius (inside a cubicle), at a speed of approximately $0.3 \mathrm{~m} / \mathrm{s}$,

- time-variant double-mobile measurements, where $4 \mathrm{Tx}$ antennas (labeled as 2, 4, 6 and 8) and $4 \mathrm{Rx}$ antennas (labeled as 1, 3, 5 and 7) were moved randomly within a $2 \mathrm{~m}$ radius (inside a cubicle), again at a speed of approximately $0.3 \mathrm{~m} / \mathrm{s}$.

Each of these scenarios was measured twice to increase the amount of collected data, and to allow for excluding possible measurement artifacts.

\section{B. Measurement Equipment}

The measurements were taken by means of the RUSK Stanford channel sounder at a center frequency of $2.45 \mathrm{GHz}$ with a bandwidth of $240 \mathrm{MHz}$, and a test signal length of 3.2 $\mu s$. The output of the sounder is the transfer function at each time instant, each frequency tone being separated by 312.5 $\mathrm{kHz}$. Owing to occasional interference (e.g. from WiFi or WiMAX equipments as well as microwave ovens), the channel characterization is actually carried out over the lower $70 \mathrm{MHz}$ of the measured spectrum, i.e. the band from 2.33 to 2.40 $\mathrm{GHz}$, yielding an initial number of tones equal to 225 .

Additionally, a total of 25 frequency tones had to be removed (approximately 4 to 5 tones every $10 \mathrm{MHz}$ ), as they were perturbed by narrowband interference caused by the antenna unequal return loss. Since all evaluations were done using the frequency domain (without carrying out Inverse Fourier Transforms into the delay domain), the cutting of frequencies does not have any impact on the channel characterization. This results effectively in $F=200$ frequency tones quasi uniformly spread over the $70 \mathrm{MHz}$ bandwidth.

The transmitter output power of the sounder was $3.2 \mathrm{~W}$. A rubidium reference in the transmit $(\mathrm{Tx})$ and receive $(\mathrm{Rx})$ units ensured accurate timing and clock synchronization. The sounder used fast $1 \times 8$ switches at both transmitter and receiver, enabling switched-array MIMO channel measurements of up to $8 \times 8$ antennas, i.e. 64 links. The Rx sensitivity was $-90 \mathrm{dBm}$. One measurement of the whole MIMO channel at one time instant is denoted as a block.

The recorded frequency responses of the MIMO channels are organized in a multi-dimensional array $H[t, f, n, m]$, with dimensions time (in blocks), frequency tone, receivers, and transmitters. We had slightly different configurations for the three different scenarios, summarized in Table I.

\section{General Concepts of Data Analysis}

Throughout this paper, the channel coefficients are considered to reflect the superposition of the following propagation effects, when expressing the channel in logarithmic scale $(\mathrm{dB})$ :

$$
\begin{array}{r}
\text { channel }=\text { path loss }+ \text { static shadowing }+ \\
+ \text { dynamic shadowing }+ \text { fading }
\end{array}
$$


TABLE I

MEASUREMENT PARAMETERS USED IN THE EVALUATION

\begin{tabular}{|c|c|c|c|}
\hline & O2I & I2I (static) & I2I (mobile) \\
\hline \hline bandwidth & \multicolumn{3}{|c|}{$2.33-2.40 \mathrm{GHz}$} \\
\hline number of frequency tones $(F)$ & \multicolumn{3}{|c|}{200} \\
\hline number of Tx antennas $(M)$ & 2 dual-polarized & 8 & 8 \\
\hline number of Rx antennas $(N)$ & 8 & 8 & 8 \\
\hline number of blocks recorded $(T)$ & 120 & 120 & 1200 \\
\hline gap between blocks & $250 \mathrm{~ms}$ & $250 \mathrm{~ms}$ & $9.83 \mathrm{~ms}$ \\
\hline recording time & $32 \mathrm{~s}$ & $32 \mathrm{~s}$ & $19.7 \mathrm{~s}$ \\
\hline
\end{tabular}

The path-loss denoted as $\Lambda$ in $\mathrm{dB}$ scale is classically defined as the deterministic distance dependence of the received power. Similarly, shadowing, denoted as $S$ in dB scale, is usually caused by obstruction of the link, and results in the individual path loss to vary with location and time.

In the experimental data, a preliminary analysis showed that shadowing is not zero-mean over time, but contains a constant part. Accordingly, shadowing is therefore expressed by the addition of two terms, i.e. static and dynamic shadowing.

Static shadowing, denoted as $\bar{S}[\mathrm{~dB}]$, is the time-invariant mean shadowing (when expressed in $\mathrm{dB}$ ) and estimated for a given link as the difference between the time-averaged received power predicted by the deterministic path loss dependence (for the same range), and the time-averaged received power on the considered link. For mobile scenarios, it is therefore related to time-invariant obstructions of the link, which can of course differ for each node. For nomadic scenarios, static shadowing has also an additional interpretation. On top of time-invariant obstruction-related shadowing, we have to take into account the constructive or destructive combination of coherent multipaths, such as reflections and diffractions on walls. In that sense, this part of static shadowing, which only exists for static links, has the same origin as spatial, or equivalently, frequency-selective fading. Since this contribution does not lead to any temporal variation, we have decided to include it under the shadowing contribution, rather than into fading. This is a particularity of nomadic links, where temporal and spatial (or frequency) fading behaviors are unrelated as they are caused by different mechanisms. Therefore, they must be modeled on a separate basis, by contrast to mobile scenarios, where static shadowing only represents the classical timeinvariant obstruction of the link (e.g. by fixed furniture or static people), and is thus frequency/space invariant. Subsequently, static shadowing is thereby written as the sum of two terms, i.e. $\bar{S}=\bar{S}_{o}+\bar{S}_{s}$, where the former is the frequency-invariant (or space-invariant) obstruction loss, and the latter is the space/frequency selective fading term which only exists for nomadic channels.

It will also be shown later in the paper that the concept of obstruction-related static shadowing is comparable with the decomposition used by well-known outdoor-to-indoor models such as the COST 231 multi-wall transmission model [22]. Although the model parameters derived in this paper are sitespecific, this is not the case of the model formalism, including the separation into static and dynamic shadowing. In particular, our static shadowing model may be replaced in a modular fashion with the corresponding model in COST 231, while keeping the other parts of our model untouched.

When path-loss and static shadowing are removed, the channel becomes at each node a zero-mean variable (in $\mathrm{dB}$ ) over time. The temporal variations are either dynamic shadowing or fading, depending on the involved mechanism, i.e. on the rate of change. Fading, denoted as $r$ or $r(t)$ in linear scale, and as $R=-20 \log _{1} 0(r)$ in $\mathrm{dB}$, is the classical small-scale fading behavior of the channel caused by multipath interference resulting from the small-scale motions of the stations and/or the environment. When fading is averaged out, the remaining variations are due to dynamic shadowing. Represented by the variable $\tilde{S}(t)[\mathrm{dB}]$, it consists in the slow temporal variation of the static loss around its static mean caused by the mobility of scatterers such as people, or of the stations themselves.

For a given link, the instantaneous loss at time $t$ is therefore proportional to $\left[R(t)+\Lambda+\bar{S}_{o}+\bar{S}_{s}+\tilde{S}(t)\right]$.

Our modeling process is as follows:

1) pre-process the data in order to represent the four effects, respectively,

2) analyze the data and, by visual inspection, propose various statistical models which might fit the data,

3) derive estimators to extract the model parameters from these data,

4) apply these estimators to the three considered environments: (i) outdoor-to-indoor static distributed nodes, (ii) indoor-to-indoor static distributed nodes, and (iii) indoor-to-indoor moving distributed nodes,

5) choose the best-fitting statistical models among those tested, and build the final model.

\section{Parameter Estimation}

This section details the data processing that is used to characterize the channels.

\section{A. Data Preprocessing}

For static measurements, temporal and frequency-selective fading are caused by different mechanisms, as explained above. Hence, the whole frequency band is first partitioned into subbands of $F_{\text {sub }}=5$ frequency tones each, over which the channel is frequency-flat. This leads to a total number of $B=F / F_{\text {sub }}$ subbands per time instant and link.

For moving measurements, time-, space- and frequencyselective fading share the same cause [23]: the motion of the node causes phase shifts in each multipath. Hence the fading 
statistics over time at a given frequency are similar to the statistics over frequency at any given time. As a consequence, it is not necessary to treat time and frequency on a separate basis and to divide the whole frequency band, which is then considered as a single subband (i.e. $F_{\text {sub }}=F$ ).

Regarding the indoor data, it was mentioned in Section II that two types of WiFi antennas were used. Hence, an antenna gain correction is implemented to compensate for the gain difference, so that the path losses can be rightfully compared with each other.

\section{B. Path Loss and Static Shadowing}

Let us consider the link between transmitter $m$ and receiver $n$. We denote by $d=d_{n m}$ the distance between these nodes. The average received power in the $b^{\text {th }}$ subband is then obtained by averaging the power within the subband and over all time samples,

$$
P[b, n, m]=\frac{1}{F_{\mathrm{sub}} T} \sum_{t=1}^{T} \sum_{f=1+(b-1) F_{\mathrm{sub}}}^{b F_{\mathrm{sub}}}|H[t, f, n, m]|^{2},
$$

with $b=1 \ldots B, n=1 \ldots N, m=1 \ldots M$ (remember that there is only one subband in the moving case, equal to the entire $70 \mathrm{MHz}$ bandwidth).

We model path-loss and static shadowing by expressing the received power $\left.P\right|_{\mathrm{dB}}$ at a distance $d$ from the transmitter as

$$
\left.P\right|_{\mathrm{dB}}(d)=\left.P_{0}\right|_{\mathrm{dB}}-\eta \cdot 10 \log _{10}\left(\frac{d}{d_{0}}\right)-\bar{S},
$$

where $P_{0}$ and $d_{0}$ denote the reference power and reference distance, respectively. For I2I scenarios, $d_{0}$ is classically fixed

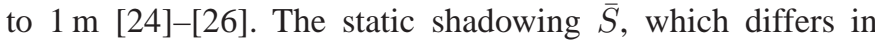
each subband in the nomadic case ${ }^{1}$ owing to the spatial fading term $\bar{S}_{s}$, is then defined as the difference between the observed power and the deterministic received power $\left.P_{0}\right|_{\mathrm{dB}}-\eta \cdot 10 \log _{10}\left(d / d_{0}\right)$. It is a time-invariant random variable for each link and each considered subband. We define the individual path-loss $L$ as

$$
L=\Lambda_{0}+\eta \cdot 10 \log _{10}\left(\frac{d}{d_{0}}\right)+\bar{S},
$$

where $\Lambda_{0}$ is the deterministic path-loss at a reference distance $d_{0}$. The path-loss and shadowing model parameters are the so-called path loss exponent, $\eta$, and the parameters describing the distributions of $\bar{S}_{o}$ and $\bar{S}_{s}$. The path loss exponent $\eta$ is common for all links and all subbands, and is estimated by a least-square fitting using the values of $P[b, n, m]$. The obstruction loss $\bar{S}_{O}$ is obtained for each link by averaging $\bar{S}$ over all frequency subbands, and its distribution is found to be lognormal, i.e. characterized by the mean and standard deviation $\mu_{\bar{S}_{o}}$ and $\sigma_{\bar{S}_{o}}$. Suitable distributions of $\bar{S}_{s}$ are the same as those investigated to model temporal fading in mobile scenarios (see Section IV-D), since spatial shadowing is caused by the same mechanisms. Finally, to simplify further notations, let us introduce the reference individual path loss as $L_{0}=$ $\Lambda_{0}+\mu_{\bar{S}_{o}}$.

\footnotetext{
${ }^{1}$ This is not the case in the mobile scenarios $\left(\bar{S}_{s}=0\right)$, which is why the full bandwidth is considered as a single subband in these cases.
}

\section{Dynamic Shadowing}

To estimate the time-variant dynamic shadowing, we first average the received power over frequency for each time instant and link. Subsequently, we further average over the small-scale fading by using a moving window spanning $T_{\mathrm{av}}=$ $2.6 \mathrm{~s}$, corresponding to either 10 samples for the static measurements or 160 samples for the moving measurements.

This yields

$$
\begin{aligned}
& P_{s}[t, b, n, m]= \\
& \frac{1}{F_{\mathrm{sub}} T_{\mathrm{av}}} \sum_{t^{\prime}=t-T_{\mathrm{av}} / 2}^{t+T_{\mathrm{av}} / 2-1} \sum_{f=1+(b-1) F_{\text {sub }}}^{b F_{\text {sub }}}\left|H\left[t^{\prime}, f, n, m\right]\right|^{2} .
\end{aligned}
$$

The choice of $T_{\mathrm{av}}$ is such that the small-scale fading is averaged out, while still following the slow variations induced by the motion of people, or by stations moving in the environment. In mobile scenarios, the window span is roughly equivalent to seven wavelengths. Such correspondence cannot be established in the nomadic case, but it should be remembered that fading is very limited in such scenarios, so that 10 samples are largely sufficient to remove any fading.

Finally, we obtain the dynamic shadowing $\tilde{S}[t, b, n, m]$ as the variation of $\left.P_{s}\right|_{\mathrm{dB}}$ around its mean,

$$
\tilde{S}=-\left[\left.P_{s}\right|_{\mathrm{dB}}-\mathrm{E}\left\{\left.P_{s}\right|_{\mathrm{dB}}\right\}\right],
$$

where the operator $\left.\right|_{\mathrm{dB}}$ denotes the conversion to $\mathrm{dB}, \mathrm{E}\{\cdot\}$ denotes the expectation over the time axis, and the $[t, b, n, m]$ dependence is dropped to simplify the notations.

A first inspection of the data reveals that dynamic shadowing is frequency-independent and log-normally distributed, in agreement with previous results [24]. In other words, $\tilde{S}$ is Gaussian distributed, with a mean $\mu_{\tilde{S}}=0$ by definition. The shadowing auto-correlation is also found to follow a negative exponential. The model parameters are therefore the standard deviation $\sigma_{\tilde{S}}$, estimated using the sample variance [27], and the slope $\tau$ of the temporal auto-correlation,

$$
R_{\tilde{S}}(\Delta t)=\frac{\mathrm{E}\{\tilde{S}(t) \tilde{S}(t+\Delta t)\}}{\sigma_{\tilde{S}}^{2}}=\mathrm{e}^{-|\Delta t| / \tau} .
$$

For mobile links, the slope $\tau$ may also be expressed as the ratio of a decorrelation distance $d_{c}$ to an effective speed $v$, corresponding to the motion of the nodes.

An important aspect of distributed or cooperative channels is that dynamic shadowing may be highly correlated between different links, which significantly affects the performance. We estimated the correlation coefficients between links $(n, m)$ and $\left(n^{\prime}, m^{\prime}\right)$ by

$$
\begin{aligned}
& C\left[b, n, m, n^{\prime}, m^{\prime}\right]= \\
& \frac{\sum_{t=1}^{T} \tilde{S}[t, b, n, m] \tilde{S}\left[t, b, n^{\prime}, m^{\prime}\right]}{\sqrt{\sum_{t=1}^{T} \tilde{S}[t, b, n, m]^{2} \sum_{t=1}^{T} \tilde{S}\left[t, b, n^{\prime}, m^{\prime}\right]^{2}}}
\end{aligned}
$$

These correlation coefficients are evaluated between all $N M$ links in one measurement. The resulting $N M(N M-1) / 2$ correlation values are then grouped in different sets:

1) links with a common $\mathrm{Rx}$ (denoted as 'Rx'),

2) links with a common Tx (denoted as 'Tx'), 
3) links with a common $\mathrm{Rx}$ or a common $\mathrm{Tx}$ (union of sets 1 and 2 , denoted as 'Rx-Tx'),

4) links with no node in common (complement of set 3 , denoted as 'disjoint'),

5) all links (union of sets 3 and 4, denoted as 'all').

For every such set, we then calculated the mean, the standard deviation, the minimum, and the maximum value of the correlation coefficients.

\section{Fading}

Small-scale fading is described by the statistics of the received signal amplitude, $r$. In our environment, we expect all kinds of fading, i.e. Ricean fading for static links, and a smooth transition from Ricean/Rayleigh fading down to Double-Rayleigh fading for mobile links. A mathematically convenient method to approximate all three distributions with certain limitations - is by using the Nakagami distribution.

Before estimating the different kinds of fading, we remove the effects of path-loss and shadow fading by normalizing each channel by its respective power as

$$
G[t, f, n, m]=\frac{H[t, f, n, m]}{\sqrt{P_{s}\left[t,\left\lceil f / F_{\text {sub }}\right\rceil, n, m\right]}},
$$

where $\lceil\cdot\rceil$ is the ceiling function. The signal amplitude is then simply defined as $r=|G|$.

To estimate the statistics of $r$, we use as ensembles the data from all tones in each subband, and all time samples. For the nomadic scenarios, we have therefore model parameter estimates for each of the considered subbands. Theoretically, one should also consider the fading correlation between different links. However, we found that this correlation was practically zero in all cases, owing to the large separation between antennas.

In the following, we discuss the different types of fading and present their parameter estimators.

1) Ricean fading: We adopt the formulation of the Ricean distribution from [28] as

$$
p_{\text {Rice }}(r)=\frac{r}{\sigma^{2}} \mathrm{e}^{-\left(\frac{r^{2}}{2 \sigma^{2}}+K\right)} \mathrm{I}_{0}\left(r \frac{\sqrt{2 K}}{\sigma}\right),
$$

where $\mathrm{I}_{0}(\cdot)$ denotes the modified Bessel function of the first kind and zero-th order, $2 \sigma^{2}$ denotes the average power of the non-coherent part, and the K-factor describes the ratio between the powers of the coherent part and the non-coherent part of the channel.

Both $K$ and $\sigma^{2}$ are estimated for every combination of $\mathrm{Rx}$ and $\mathrm{Tx}$ by numerical curve fitting to the cumulative distribution function (cdf) of the Ricean distribution. Note that if $\mathrm{E}\left\{r^{2}\right\}=1, \sigma$ and $K$ follow the relationship: $2 \sigma^{2}=1 /(K+1)$.

2) Second-order scattering fading distribution: The Ricean fading distribution includes pure Rayleigh fading as the limiting case for $K=0$. In some measured scenarios, however, we observe fading that is more severe than Rayleigh fading. To model this effect, we assume that the channel can be expressed as $G=w_{0} e^{j \theta}+w_{1} G_{1}+w_{2} G_{2} G_{3}$, where $G_{1}, G_{2}, G_{3}$ are i.i.d. complex normal random variables with zero mean and unit variance, and $\theta$ is a constant phase angle from $[0,2 \pi]$. The three terms can be interpreted as a line-of-sight component, a single-bounce Rayleigh-fading component and a two-bounce Double-Rayleigh-fading component, respectively. The weighting factors $w_{0}, w_{1}, w_{2}>0$ determine the relative powers of the three components. The probability density function of $r=|G|$ is then given as shown in [20], [29] by the so-called secondorder scattering fading (SOSF) distribution,

$$
p_{\text {SOSF }}(r)=r \int_{0}^{\infty} \omega \mathrm{e}^{-w_{1}^{2} \omega^{2} / 4} \frac{4 J_{0}(r \omega) J_{0}\left(w_{0} \omega\right)}{4+w_{2}^{2} \omega^{2}} \mathrm{~d} \omega,
$$

where $\mathrm{J}_{0}$ is the Bessel function of the first kind and zero-th order. Note that $\mathrm{E}\left\{r^{2}\right\}=1$ is achieved when $w_{0}^{2}+w_{1}^{2}+w_{2}^{2}=1$ so that the distribution can be specified by two parameters [20],

$$
\begin{aligned}
& \alpha=\frac{w_{2}^{2}}{w_{0}^{2}+w_{1}^{2}+w_{2}^{2}}, \\
& \beta=\frac{w_{0}^{2}}{w_{0}^{2}+w_{1}^{2}+w_{2}^{2}},
\end{aligned}
$$

where $(\alpha, \beta)$ are constrained to the triangle $\alpha \geq 0, \beta \geq 0$, $\alpha+\beta \leq 1$.

The SOSF distribution naturally encompasses Ricean fading ( $\alpha=0$, with $\beta /(1-\beta)$ being the $\mathrm{K}$-factor), Rayleigh fading ( $\alpha=\beta=0)$, as well as Double-Rayleigh fading $(\alpha=1$, $\beta=0)$.

Assuming for the moment that $w_{0}=0$, the remaining parameters $w_{1}, w_{2}$ can be estimated based on the method of moments [29] as $\hat{w}_{2}=\sqrt[4]{S_{4} / 2-S_{2}^{2}}$ and $\hat{w}_{1}=\sqrt{S_{2}-\hat{w}_{2}^{2}}$, where $S_{i}$ is the $i$ th sample moment. In our data analysis, we use these estimates with $\hat{w}_{0}=0$ as starting point for a CDF curve fitting of the SOSF distribution to the empirical CDFs.

3) Nakagami fading: The Nakagami- $m$ distribution is given by [24]:

$$
p_{\text {Naka }}(r)=\frac{2}{\Gamma(m)}\left(\frac{m}{\Omega}\right)^{m} r^{2 m-1} \mathrm{e}^{-m r^{2} / \Omega},
$$

where $\Omega$ is the second moment, $\Gamma(\cdot)$ denotes the Gamma function and the m-parameter (sometimes known as the shape parameter) is defined as $m=\Omega^{2} / \mathrm{E}\left\{\left(r^{2}-\Omega\right)^{2}\right\}, m \geq 0.5$.

The second moment $\Omega$ can be estimated by the unbiased maximum-likelihood estimator $\hat{\Omega}=S_{2}$, while the estimator of the m-parameter is the approximation of the maximumlikelihood estimator proposed in [30]. Note that $\Omega=1$ if $\mathrm{E}\left\{r^{2}\right\}=1$.

While the Nakagami distribution is mathematically tractable for analytical investigations, it has a number of shortcomings: (i) in contrast to the Ricean and Rayleigh/Double-Rayleigh distributions, it has no physical interpretation, (ii) for this reason, it does not fit the measurements as well, (iii) there is no analytical random-number generator for this distribution (only slow, iterative methods exist).

\section{Channel Characterization Results}

\section{A. Outdoor-to-Indoor Channels}

The parameters of the Outdoor-to-Indoor (O2I) environment were extracted from all channels between the 2 (dualpolarized) $\mathrm{Tx}$ antennas and all $12 \mathrm{Rx}$ locations (thereby 


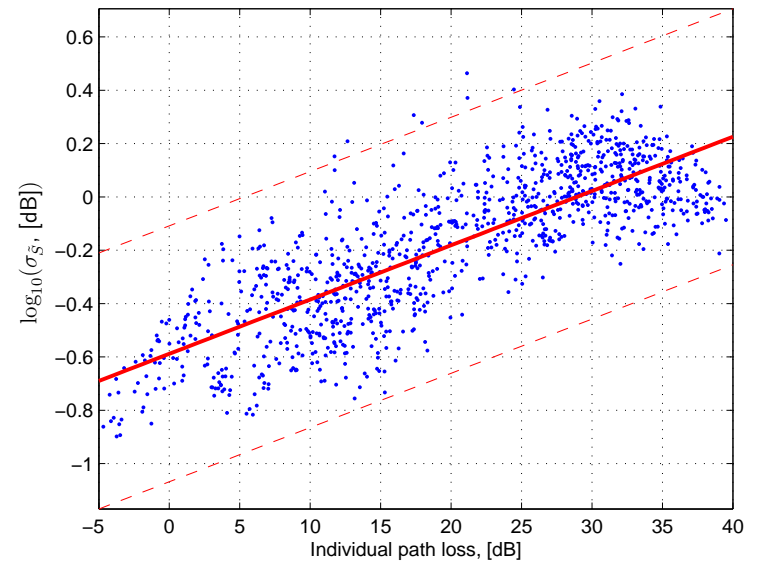

Fig. 3. Standard deviation of dynamic shadowing for the O2I scenario (static receivers)

including the relay nodes). The parameter estimation was carried out as described in Section IV.

1) Path loss and static shadowing: The individual path loss of all $12 \mathrm{Rx}$ nodes is calculated relative to the individual path loss from BS antenna 1 to relay 2. This reference link corresponds to the shortest range, and also to the best reception point in the room, i.e. at the closest wall. Unfortunately, the penetration loss into the building, represented by $\mu_{\bar{S}_{o}}$ in our notation, cannot be extracted from the available measurement data. Additionally, only twelve O2I links were measured (by contrast to 64 in the I2I case), implying a reduced number of data points. Furthermore, a preliminary analysis also shows that $\mu_{\bar{S}_{o}}$ differs between the nodes on the right and left sides of the building, owing to the shadowing effect of building 2 for six of the left-side nodes ( $\operatorname{Rx}$ nodes 1, 4, 7 and 8, and relay nodes 3 and 5). For these three reasons, it was decided not to estimate $\eta$ and the statistics of $\bar{S}$ in (2). However, we will show later that our decomposition into path-loss and static shadowing enables to use existing outdoor-to-indoor path loss models to overcome this lack of measurements.

2) Dynamic shadowing: Dynamic shadowing $\tilde{S}$ is lognormal distributed, so we consider its standard deviation, $\sigma_{\tilde{S}}$, which is strongly correlated with the path loss in nomadic scenarios. Fig. 3 demonstrates that the larger the path loss, the larger the dynamic shadowing variance becomes. We model this correlation using an exponential fit

$$
\log _{10}\left(\sigma_{\tilde{S}}\right)=\log _{10}\left(\sigma_{s, 0}\right)+0.02\left(L-L_{0}\right)+\sigma_{\sigma_{\tilde{S}}},
$$

where $\sigma_{s, 0}=0.27 \mathrm{~dB}$, and $\sigma_{\sigma_{\tilde{S}}}$ is a zero-mean Gaussian distributed random variable with a standard deviation of 0.16 . The slope of the temporal auto-correlation is estimated as $\tau=1.74 \mathrm{~s}$.

Regarding the correlation coefficients of the dynamic shadowing, the mean, the standard deviation, the minimum, and the maximum value of all different subsets (cf. Sec. IV-C) are given in Table II. It can be seen that very high shadow fading correlations as well as anti-correlations occur in the measurements. In the O2I case, the distribution of shadow
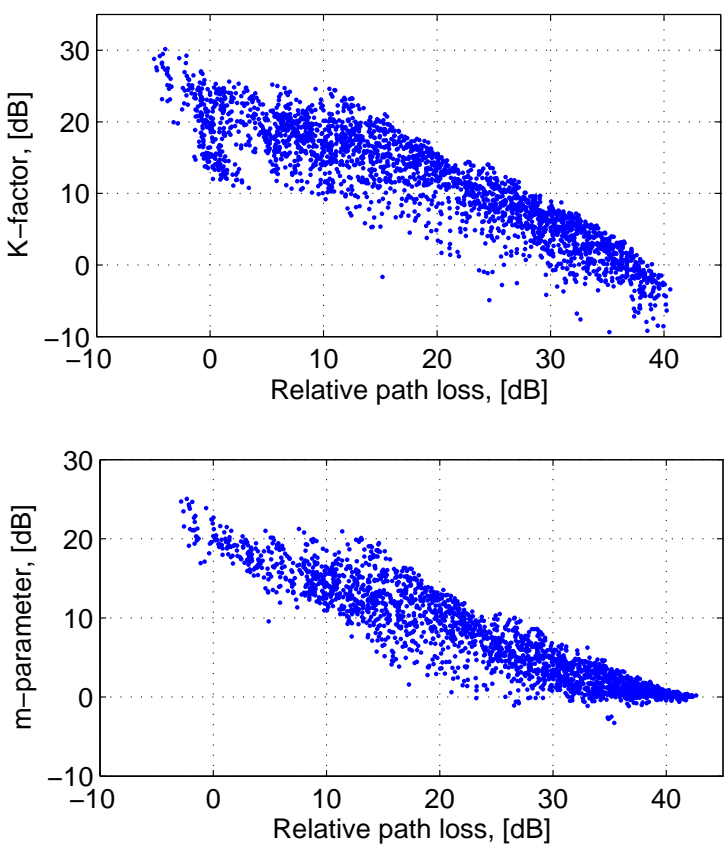

Fig. 4. K-factor and m-parameter vs. distance for the O2I nomadic data

fading correlations follows a Gaussian distribution quite well.

3) Fading: Given the facts that $\mathrm{Tx}$ and $\mathrm{Rx}$ nodes are all static and randomness was only introduced by people moving in the building, the temporal fading statistics are expected to be strongly Ricean. Our analysis indeed reveals that the Kfactor is generally very high, and decreases with increasing path loss (see Fig. 4).

The variation vs. path loss is well fitted by

$$
\left.K\right|_{\mathrm{dB}}=\left.K_{0}\right|_{\mathrm{dB}}-0.60\left(L-L_{0}\right)+\sigma_{K},
$$

where $\left.K\right|_{\mathrm{dB}}$ is the Ricean $\mathrm{K}$-factor expressed in $\mathrm{dB}, \sigma_{K}$ is a random variable (approximately Gaussian) of standard deviation equal to $3.8 \mathrm{~dB}$, and $\left.K_{0}\right|_{\mathrm{dB}}$ is equal to $22.5 \mathrm{~dB}$ in our experiment. Alternative models of $\left.K_{0}\right|_{\mathrm{dB}}$ can be found in [9] for fixed outdoor-to-outdoor channels. Note however that the decrease rate in (15) is estimated as $0.24 \mathrm{~dB} / \mathrm{dB}$ in [9]. This value is smaller than our own decrease rate, but once again, we stress that our measured path loss includes a large shadowing by neighboring buildings. This explains the discrepancy.

When fitting the Nakagami distribution to the data, also large m-parameters are observed, and the values are consistent with the observed K-factors, which is due to the close match of the Nakagami distribution to the Ricean distribution for large values of the m-parameter. For $m=1$, the Nakagami distribution is equal to Rayleigh fading; for values $0.5<m<$ 1 , the Nakagami distribution resembles fading that is worse than Rayleigh (i.e. favoring smaller amplitude values). Note that because the m-parameter is lower-bounded by 0.5 , the apparent standard deviation of $m$ as a function of path-loss naturally decreases with increasing path-loss. In addition, fit values of $m$ very close to $-3 \mathrm{~dB}$ are relatively rare. Instead, $m$ tends to concentrate around $0 \mathrm{~dB}$ for large path loss levels. 
TABLE II

SHADOWING CORRELATION STATISTICS IN ALL SCENARIOS

\begin{tabular}{|c|c|c|c|c|c|}
\hline Scenario & Subset & Mean & Std. & Max & Min \\
\hline \hline O2I & all & 0.02 & 0.31 & 0.98 & -0.89 \\
\hline \hline \multirow{4}{*}{$\begin{array}{c}\text { I2I } \\
\text { static }\end{array}$} & all & 0.00 & 0.27 & 0.94 & -0.90 \\
\cline { 2 - 6 } & Rx & 0.02 & 0.30 & 0.93 & -0.90 \\
\cline { 2 - 6 } & Tx & 0.01 & 0.30 & 0.94 & -0.89 \\
\cline { 2 - 6 } & Rx-Tx & 0.01 & 0.30 & 0.94 & -0.90 \\
\cline { 2 - 6 } & disjoint & 0.00 & 0.27 & 0.90 & -0.88 \\
\hline \hline \multirow{4}{*}{$\begin{array}{c}\text { I2I } \\
\text { double } \\
\text { mobile }\end{array}$} & all & 0.16 & 0.39 & 0.97 & -0.90 \\
\cline { 2 - 6 } & Rx & 0.29 & 0.36 & 0.89 & -0.40 \\
\cline { 2 - 6 } & Tx & 0.29 & 0.42 & 0.97 & -0.74 \\
\cline { 2 - 6 } & Rx-Tx & 0.29 & 0.39 & 0.97 & -0.74 \\
\cline { 2 - 6 } & disjoint & 0.06 & 0.36 & 0.81 & -0.90 \\
\hline \hline \multirow{4}{*}{$\begin{array}{c}\text { I2I } \\
\text { mobile } \\
\text { mobile }\end{array}$} & all & 0.05 & 0.38 & 0.99 & -0.91 \\
\cline { 2 - 6 } & Rx & 0.47 & 0.41 & 0.99 & -0.79 \\
\cline { 2 - 6 } & Tx & -0.01 & 0.35 & 0.80 & -0.83 \\
\cline { 2 - 6 } & Rx-Tx & 0.23 & 0.45 & 0.99 & -0.83 \\
\cline { 2 - 6 } & disjoint & 0.00 & 0.34 & 0.90 & -0.91 \\
\hline \hline \multirow{2}{*}{$\begin{array}{c}\text { I2I } \\
\text { single } \\
\text { mobile } \\
(\text { Tx })\end{array}$} & all & 0.05 & 0.41 & 0.99 & -0.93 \\
\cline { 2 - 6 } & Rx & 0.00 & 0.36 & 0.81 & -0.82 \\
\cline { 2 - 6 } & Tx & 0.37 & 0.50 & 0.99 & -0.84 \\
\cline { 2 - 6 } & Rx-Tx & 0.19 & 0.47 & 0.99 & -0.84 \\
\cline { 2 - 6 } & disjoint & 0.01 & 0.37 & 0.87 & -0.93 \\
\hline
\end{tabular}

Still, for the largest part of the range of path loss values, the variation of the m-parameter vs. path loss is fitted by

$$
\log _{10}(m)=\log _{10}\left(m_{0}\right)-0.052\left(L-L_{0}\right)+\sigma_{m}, m \geq 0.5
$$

where $\sigma_{m}$ is a random variable (approximately Gaussian) of standard deviation equal to 0.40 , and $\log _{10}\left(m_{0}\right)=1.88$ in our case. As a consequence of the apparent reduction of standard deviation at high path loss, this model is not rigorously valid for larger values of path-loss. However, practically, it can still be used in combination with a rejection method, i.e. drawing a candidate for $\sigma_{m}$, verifying that the generated $m$ meets the constraint, and if not met, drawing another candidate until it is fulfilled.

\section{B. Indoor-to-Indoor Channels for Static Nodes}

If not indicated differently, the parameters from the indoorto-indoor channels were extracted from the distributed-nodes environment shown in Fig. 2, for all channels between the 8 transmitters and 8 receivers. The parameter estimation was carried out as described in Section IV.

1) Path loss and shadowing: We evaluated the relative path loss as a function of the Tx-Rx distance from the data, as highlighted in Fig. 5. From the graph, we extract the path-loss model as

$$
L=L_{0}+1.75 \cdot 10 \log _{10}\left(\frac{d}{d_{0}}\right)+\bar{S}_{o}+\bar{S}_{s},
$$

where $d_{0}=1 \mathrm{~m}$ and $\bar{S}_{s}=-20 \log _{1} 0\left(\bar{s}_{s}\right)$. We further implicitly assume that $\mu_{\bar{S}_{o}}=0$, i.e. that the reference individual path loss $L_{0}$ is equal to the deterministic reference path loss

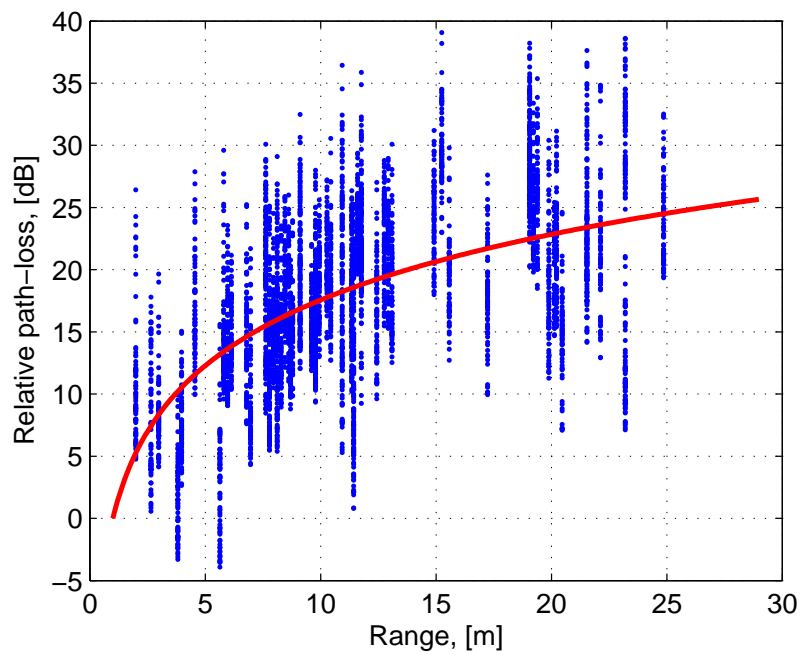

Fig. 5. Relative path loss vs. distance and linear fit of (17)

$\Lambda_{0}$ indoors (this results from the fact that $d_{0}=1 \mathrm{~m}$ by contrast to the O2I case). Interestingly, the value of $\eta=1.75$ is smaller than 2 , which tends to indicate that waveguiding propagation effects take place. The obstruction loss $\bar{S}_{o}$ is found to be a zero-mean Gaussian distributed variable, with a standard deviation $\sigma_{\bar{S}_{\circ}}=4.43 \mathrm{~dB}$.

The statistics of the spatial fading part included in static shadowing for this scenario vary between Ricean fading, pure Rayleigh fading and worse-than-Rayleigh fading, i.e., fading in which smaller amplitudes are more probable than in the Rayleigh distribution. For some links, the distribution of $\bar{s}_{s}$ even approaches the Double-Rayleigh distribution. As outlined in Section IV-D, a smooth transition between Ricean and below-Rayleigh distributions can be modeled by both the Nakagami distribution $p_{\text {Naka }}$ (with $0.5<m<1$ ) and the SOSF distribution $p_{\text {SOSF }}$. Consequently, we fit these two distributions to the measurement data, by normalizing the data to satisfy $\mathrm{E}\left\{|G|^{2}\right\}=1$ and forcing both distributions to obey this constraint, i.e. we choose $\Omega=1$ for Nakagami and $w_{0}^{2}+w_{1}^{2}+w_{2}^{2}=1$ for SOSF. The Nakagami fitting problem then reduces to a one-dimensional optimization with respect to $m$, while the SOSF fitting problem is a two-dimensional optimization. The fitting is implemented using standard nonlinear minimization algorithms, where the $L_{\infty}$ norm of the CDF deviation plays the role of a goodness of fit measure (the smaller the norm, the better the fit). The optimization process is initialized by the moment-based or ML parameter estimates mentioned in Section IV-D.

When comparing the goodness of fit for the two distributions for all links, we find that the SOSF distribution generally achieves a better fit than Nakagami. This is expected, both because unlike Nakagami, SOSF has a physical interpretation, and SOSF allows us to tweak two parameters for fitting as opposed to one in the Nakagami case. The derived values of $\alpha_{s}$ and $\beta_{s}$ are depicted on Fig. 6 for all links. The triangle is the permissible parameter region, $\mathcal{T}=\left\{\left(\alpha_{s}, \beta_{s}\right) \mid \alpha_{s} \geq\right.$ $\left.0, \beta_{s} \geq 0, \alpha_{s}+\beta_{s} \leq 1\right\}$. While there is no discernible correlation between $\left(\alpha_{s}, \beta_{s}\right)$ and the individual relative path 


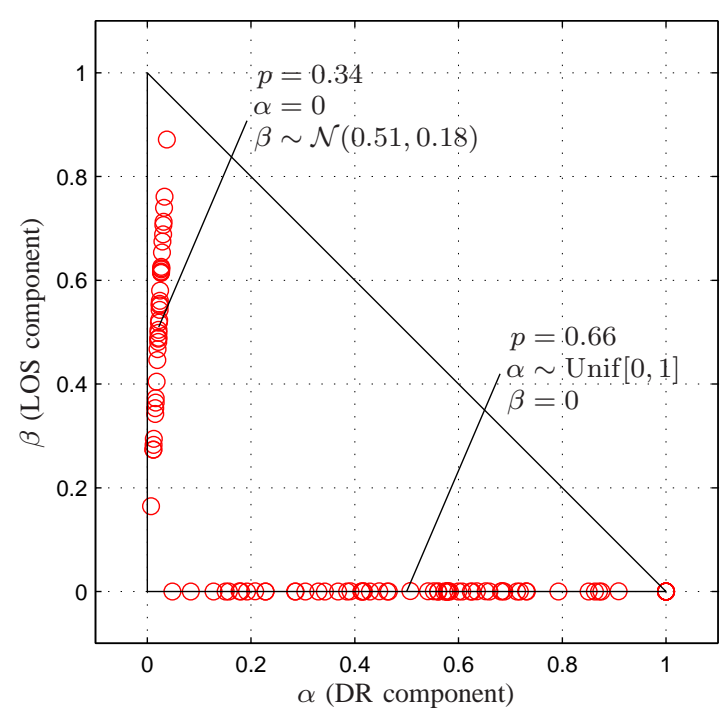

Fig. 6. SOSF fitted parameters for the distribution of spatial static shadowing

loss, the parameters are clearly distributed along the axes, so that the joint distribution of $\alpha_{s}$ and $\beta_{s}$ can be represented by the following bimodal distribution:

$$
\begin{aligned}
\left(\alpha_{s}, \beta_{s}\right) & \sim 0.34 \delta\left(\alpha_{s}\right) \cdot \mathcal{N}_{\mu=0.51, \sigma=0.18}\left(\beta_{s}\right) \\
& +0.66 \operatorname{Unif}_{[0,1]}\left(\alpha_{s}\right) \cdot \delta\left(\beta_{s}\right),
\end{aligned}
$$

constrained to the triangle $\mathcal{T}$ of permissible $\left(\alpha_{s}, \beta_{s}\right)$. Here, Unif $_{[0,1]}$ is a uniform distribution over $[0,1]$.

2) Dynamic shadowing: For the standard deviation of the dynamic shadowing, we observed the same effect as in the O2I case. It is again strongly correlated with the path loss (cf. Fig. 3) and is modeled as in (14), $\sigma_{\sigma_{\tilde{s}}}^{\prime}$ being zeromean Gaussian distributed with a standard deviation of 0.22 . Furthermore, we may express $\sigma_{\tilde{S}}$ as a function of the distance as

$$
\log _{10}\left(\sigma_{\tilde{S}}\right)=\log _{10}(1.85)+0.2 \log _{10}\left(\frac{d}{d_{0}}\right)+\sigma_{\sigma_{\tilde{S}}}^{\prime},
$$

where $\sigma_{\sigma_{\tilde{S}}}^{\prime}$ is a zero-mean Gaussian distributed random variable standard deviation of 1.13. The slope of the temporal auto-correlation is estimated as $\tau=2.5 \mathrm{~s}$.

Regarding the dynamic shadowing correlation, the results are summarized in Table II. For the static I2I scenario, it can only be noticed that all the sets show a very similar behavior. Furthermore, no clear relationship with the geometry of the links could be found.

3) Fading: For static antennas, the channel gain is naturally found to be Ricean distributed over time, with the K-factor closely related to the to the Tx-Rx distance, as illustrated in Fig. 7. This trend can be fitted by

$$
\left.K\right|_{\mathrm{dB}}=16.90-5.25 \log _{10}\left(\frac{d}{d_{0}}\right)+\sigma_{K}^{\prime},
$$

where $d$ is the Tx-Rx distance in meters, $d_{0}=1 \mathrm{~m}$ and $\sigma_{K}^{\prime}$ is approximately a random Gaussian variable of standard
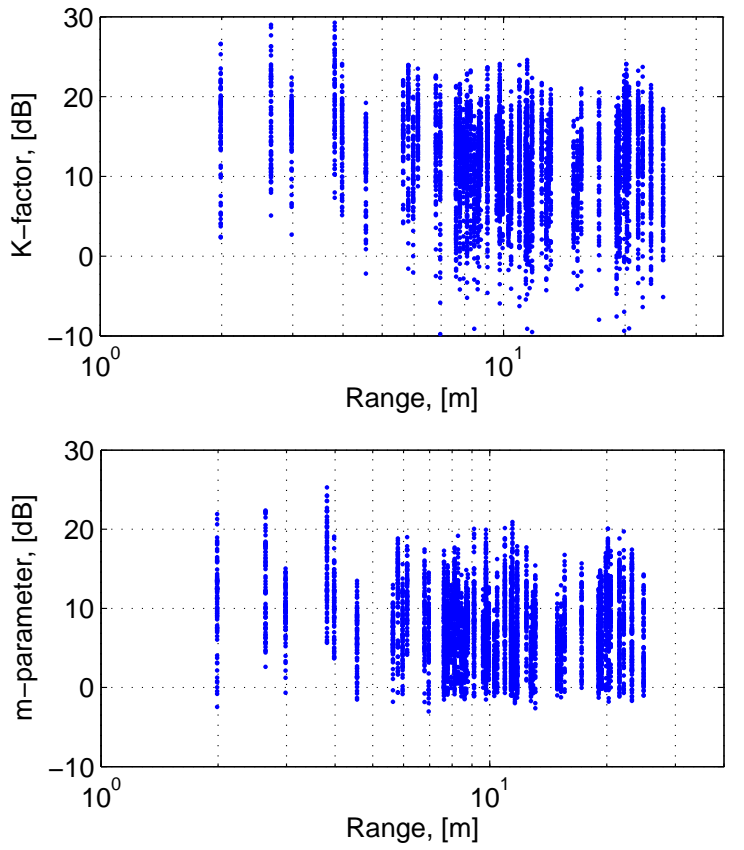

Fig. 7. K-factor and m-parameter vs. distance for the I2I nomadic data

deviation equal to $6 \mathrm{~dB}$.

A similar trend is found for the Nakagami m-parameter, which is fitted by

$$
\log _{10}(m)=1.35-0.50 \log _{10}\left(\frac{d}{d_{0}}\right)+\sigma_{m}^{\prime}
$$

over distance. Variable $\sigma_{m}^{\prime}$ is Gaussian distributed with a standard deviation equal to 0.48 . The distribution is naturally truncated so that $m>0.5$.

\section{Indoor-to-Indoor Channels for Mobile Nodes}

1) Path loss and static shadowing: Expectedly, the measured static shadowing is frequency flat for mobile nodes (so that $\bar{S}=\bar{S}_{o}$, and $\bar{S}_{s}=0$, i.e. the SOSF parameters $\left(\alpha_{s}, \beta_{s}\right)$ are equal to $(0,1)$ corresponding to a Ricean distribution with infinite $\mathrm{K}$-factor). Both the path loss exponent (estimated as 1.77 ) and the standard deviation of $\bar{S}_{o}$ (estimated as $4.6 \mathrm{~dB}$ ) are very similar to the nomadic case. In the proposed model, we will use the above values, as the estimation accuracy is expected to be higher for mobile measurements (because the number of realizations is higher).

2) Dynamic shadowing: In contrast to the nomadic case, $\sigma_{\tilde{S}}$ does not depend on the path loss any more when one or both stations are moving, but is rather constant. We observed it to be similar to that of static shadowing, hence we model $\sigma_{\tilde{S}}=$ $\sigma_{\bar{S}_{o}}$. The slope of the temporal auto-correlation is estimated as $\tau=1.5 \mathrm{~s}$ for both single- and double-mobile links, which provides an equivalent decorrelation distance $d_{c}=0.45 \mathrm{~m}$ considering that the nodes move at $0.3 \mathrm{~m} / \mathrm{s}$. Note that the absence of any difference between single- and double-mobile links regarding dynamic shadowing characteristics is probably due to the fact that dynamic shadowing is mostly caused by 


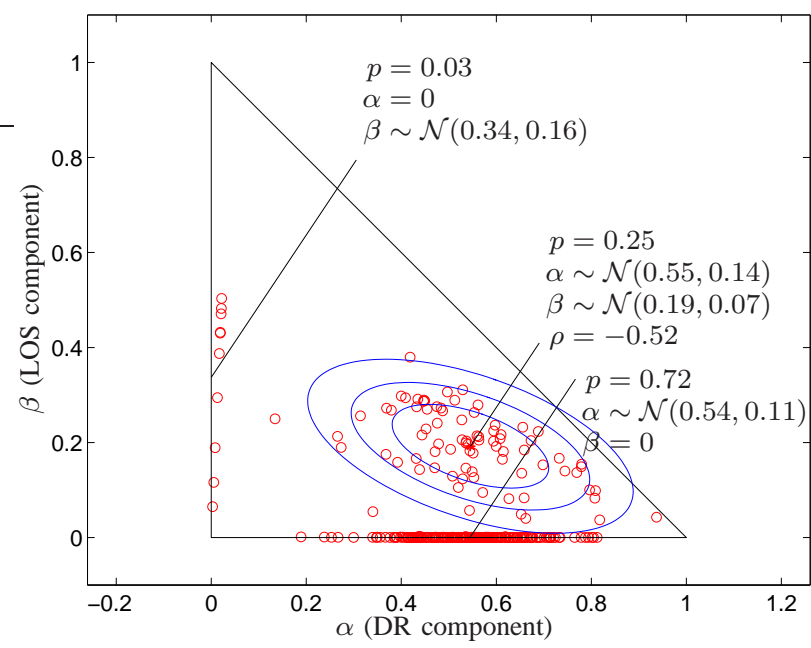

Fig. 8. SOSF fitted parameters $(\alpha, \beta)$ and multi-modal Gaussian model for double-mobile links. The ellipses contain $50 \%, 80 \%$, and $95 \%$ of all realizations, respectively, for the non-degenerate mode.

moving people obstructing the link (and only partly by the fact that the antennas might be occasionally shadowed by furniture at both ends). It is of course not possible to separate both effects, hence both the decorrelation time and distance will be considered in the model.

Table II also provides the results on the correlation coefficients. In the table, we distinguish between the two types of mobility previously defined. When at least one of the nodes is moving, it can be seen that the sets for which the moving node is common clearly show a higher correlation than the sets for which the moving node is not common to both links. As an example, for the single-mobile case with moving $\mathrm{Rx}$, the 'Rx' set shows a higher correlation than the 'Tx' set, while the opposite is observed for the the single-mobile case with moving Tx. For the double-mobile case, the 'Rx', 'Tx' and 'Rx-Tx' sets (all containing a joint moving node) show similar values, while the 'disjoint' set has a significantly lower mean. This behavior is actually quite intuitive, as the joint moving node is a source of positive correlation across the links. For the sequel, we will thus partition the set of links into two subsets: (i) links with a common moving node (the set $\mathrm{Rx}$-Tx for the double mobile case and the set Rx (resp. Tx) for the single mobile Rx (resp. Tx) case) and (ii) links with no moving node in common (the complement of (i)).

3) Fading: The small-scale fading statistics for this scenario vary between Ricean and Double-Rayleigh fading. After fitting both the Nakagami and the SOSF distributions, we find that the latter achieves a much better fit. In order to characterize the distribution parameters $(\alpha, \beta)$, we first note that there is again no discernible correlation between these parameters and the individual relative path loss (analogous to $\bar{S}_{s}$, which is not surprising). Hence, we take again a stochastic approach and distinguish between single-mobile and doublemobile links.

Fig. 8 shows the distribution parameters $(\alpha, \beta)$ for all double-mobile links. We identify three clusters in the distribution: (1) non-line-of-sight cases, $\beta \approx 0$, where the distribution is a mixture of Rayleigh and Double-Rayleigh fading, (2)
Ricean cases, $\alpha \approx 0$, and (3) cases where both $\alpha$ and $\beta$ are larger than zero. Each cluster is modeled with a bivariate Gaussian distribution, such that the overall distribution of $(\alpha, \beta)$ results in a three-modal Gaussian. The figure also shows the distribution parameters for each mode, such that the global distribution of $(\alpha, \beta)$ for double-mobile links is

$$
\begin{aligned}
(\alpha, \beta) & \sim 0.03 \delta(\alpha) \cdot \mathcal{N}_{\mu=0.34, \sigma=0.16}(\beta) \\
& +0.72 \mathcal{N}_{\mu=0.54, \sigma=0.11}(\alpha) \cdot \delta(\beta) \\
& +0.25 \mathcal{N}_{\mu=[0.55,0.19], \sigma=[0.14,0.07], \rho=-0.52}(\alpha, \beta)
\end{aligned}
$$

constrained to the triangle $\mathcal{T}$ of permissible $(\alpha, \beta)$.

For single-mobile links, similar trends are found [20], and the resulting three-modal Gaussian is then

$$
\begin{aligned}
(\alpha, \beta) & \sim 0.09 \delta(\alpha) \cdot \mathcal{N}_{\mu=0.27, \sigma=0.14}(\beta) \\
& +0.59 \mathcal{N}_{\mu=0.40, \sigma=0.14}(\alpha) \cdot \delta(\beta) \\
& +0.32 \mathcal{N}_{\mu=[0.39,0.24], \sigma=[0.12,0.09], \rho=-0.13}(\alpha, \beta),
\end{aligned}
$$

again constrained to the triangular set $\mathcal{T}$. Note that for doublemobile links, the $\alpha$ values are significantly higher than in the single-mobile case, indicating more severe fading conditions.

Finally, it is interesting to note that the first mode (with $\alpha=0$ ), is in fact Ricean-distributed, and thus an equivalent $\mathrm{K}$-factor can be computed. In our measurement, the median equivalent $\mathrm{K}$-factor (in natural scale) when $\alpha=0$ is 0.31 for single-mobile links, and 0.69 for double-mobile links, i.e. these channels are almost Rayleigh distributed.

\section{LinK-LEVEl Simulation Model}

This section integrates the results of the previous section to build channel models for both O2I and I2I scenarios. Before doing so, let us summarize the main requirements which cooperative channel models should meet:

- differentiate between the different types of mobility: nomadic, single-mobile and double-mobile, as the analysis has revealed that the channel behavior is very dependent on the mobility scenario; that implies to correctly identify which mechanisms are space-varying and/or timevarying,

- allow for integration of site-specific models, especially regarding the O2I segment, which is significantly influenced by the outdoor environment,

- include a model of dynamic shadowing correlation, as this parameter largely affects cooperative system performance,

- rely, as much as possible, and in agreement with the data, on Gaussian-based distributions (e.g. Rayleigh, Rice, lognormal, SOSF, etc.), since random generators are then easily built.

Hereafter, we describe the models for both O2I and I2I channels. Table III provides the values of the parameters used by the models. 


\section{A. Outdoor-to-Indoor Channel Model}

The narrowband complex channel $h_{n}$ from the outdoor base station to the indoor node $n$ is described by

$$
h_{n}(t)=\frac{1}{10^{L_{n} / 20} \cdot 10^{\tilde{S}_{n}(t) / 20}} \cdot g_{n}(t)
$$

where $L_{n}$ is the combination of deterministic path loss $\Lambda_{n}$ and static shadowing $\bar{S}_{n} ; \tilde{S}_{n}$ is the real-valued dynamic shadowing; and $g_{n}$ is the complex normalized fading channel. All these contributions are modeled based on the analysis of Section V-A. We summarize here the main steps required to model $h_{n}$.

1) Path loss and static shadowing: The path loss and static shadowing have not been analyzed in this paper due to the limited number of O2I scenarios. We recommend to model them by using the approaches of [22], [25], where $L_{n}$ is generally represented for LOS scenarios by

$$
L_{n}=\Lambda_{0}+\eta \cdot 10 \log _{10}\left(\frac{d_{n}}{d_{0}}\right)+\bar{S}_{n},
$$

where $\Lambda_{0}$ is the equivalent outdoor path-loss at the best wall, $d_{0}$ is the distance from the BS to the best wall, and $\eta$ is given in Table III. $\bar{S}_{n}$ is modeled as the sum of two terms, $\bar{S}_{o, n}$ and $\bar{S}_{s, n}$. The first term $\bar{S}_{o, n}$ is a Gaussian variable, whose mean is given by

$$
\mu_{\bar{S}_{o}}=L_{o 2 i}+\max \left[L_{i, 1}, L_{i, 2}\right],
$$

where $L_{o 2 i}=L_{e}+L_{g}(1-\cos \phi)^{2}$ is the outdoor-to-indoor excess path-loss, with $L_{e}$ being the path loss through the external wall at normal incidence $(\phi=0)$, and $L_{g}$ is the additional external wall loss incurred at grazing incidence ( $\phi=\pi / 2) ; L_{i, 1}$ is the indoor path loss proportional to the indoor distance,

$$
L_{i, 1}=0.5\left(d-d_{0}\right),
$$

and $L_{i, 2}=n_{w} L_{w}$ is the excess attenuation caused by $n_{w}$ internal walls. In NLOS cases, the expression is slightly modified to include a floor gain [22]. Values for the above parameters at $2.45 \mathrm{GHz}$ are detailed in [31]. Note that $\Lambda_{0}+L_{o 2 i}$ represents what we denoted as $L_{0}$ in Section V-A.

The standard deviation $\sigma_{\bar{S}_{o}}$ and the parameters describing the statistics of $\bar{S}_{s, n}=\left.\bar{s}_{s, n}\right|_{\mathrm{dB}}$ are given in Table III.

2) Dynamic shadowing: Dynamic shadowing is modeled as a zero-mean Gaussian variable, whose standard deviation is given by (14). The shadowing temporal auto-correlation is modeled as a decreasing exponential, whose decay time $\tau$ is listed in Table III. The correlation coefficient $C\left[n, n^{\prime}\right]$ between $\tilde{S}_{n}$ and $\tilde{S}_{n^{\prime}}$ is given by a truncated Gaussian distribution $\mathcal{N}_{[-1,1]}\left(\mu_{C}, \sigma_{C}\right)$, whose parameters are given in Table III. Hence, to model the dynamic shadowing $\tilde{S}_{n}(t)$ and $\tilde{S}_{n^{\prime}}(t)$ on two links over $T$ time samples $(t=[1, \ldots, T])$, we first use the following auto-regressive process to generate autocorrelated dynamic shadowing values,

$$
\begin{aligned}
& x(t)=\mathrm{e}^{-1 / \tau} x(t-1)+\sqrt{1-\mathrm{e}^{-2 / \tau}} g_{x}(t), \\
& y(t)=\mathrm{e}^{-1 / \tau} y(t-1)+\sqrt{1-\mathrm{e}^{-2 / \tau}} g_{y}(t),
\end{aligned}
$$

where $g_{x}$ and $g_{y}$ are both time series of length $T$, whose values are drawn independently form a normal distribution $\mathcal{N}(0,1)$. This ensures an autocorrelation $\mathrm{E}\{x(t) x(t+\Delta t)\}=$ $\mathrm{e}^{-|\Delta t| / \tau}$, and similar for $y(t)$. We finally generate the standard deviations $\sigma_{\tilde{S}_{n}}$ and $\sigma_{\tilde{S}_{n}}$ as a function of the respective ranges $d_{n}$ and $d_{n^{\prime}}$, and correlate both time series at each time $t$ as

$$
\begin{aligned}
& {\left[\begin{array}{c}
\tilde{S}_{n}(t) \\
\tilde{S}_{n^{\prime}}(t)
\end{array}\right]=} \\
& {\left[\begin{array}{c}
\sigma_{\tilde{S}_{n}} \\
\sigma_{\tilde{S}_{n^{\prime}}}
\end{array}\right] \odot\left[\begin{array}{cc}
1 & C\left[n, n^{\prime}\right] \\
C\left[n, n^{\prime}\right] & 1
\end{array}\right]^{1 / 2}\left[\begin{array}{l}
x(t) \\
y(t)
\end{array}\right],}
\end{aligned}
$$

where $\odot$ is the Hadamard element-wise product. Note that when multiple links are concerned, the full correlation matrix containing all correlation coefficients should satisfy a positive semi-definiteness constraint. For $N$ nodes, there are $N(N-$ 1) $/ 2$ links, so that the correlation matrix is $N(N-1) / 2 \times$ $N(N-1) / 2$ and contains the various cross-link correlation coefficients randomly generated by the model. One practical way to build a valid correlation matrix is the rejection method, i.e. drawing a candidate, verifying the constraint, and if not met, drawing another candidate until it is fulfilled.

3) Fading: The amplitude of $g_{n}$ is modeled for static Rx nodes by a Ricean distribution whose $\mathrm{K}$-factor is related to the relative path-loss $L_{n}-L_{0}$ as outlined by (15). Generating complex Ricean variables relies on using two normal variable random variables, i.e. any realization of $g_{n}$ is given by

$$
g_{n}=\sqrt{\frac{K}{K+1}} \mathrm{e}^{j \theta}+\sqrt{\frac{1}{2(K+1)}}(u+j v)
$$

where $\theta$ is a random phase (fixed over time, but different for each link), and $u$ and $v$ are normal variables $\mathcal{N}(0,1)$. The fading processes between different links are taken as uncorrelated.

\section{B. Indoor-to-Indoor Channel Model}

The narrowband complex channel $h_{n m}$ from indoor node $m$ to indoor node $n$ is described by

$$
h_{n m}(t)=\frac{1}{10^{L_{n m} / 20} \cdot 10^{\tilde{S}_{n m}(t) / 20}} \cdot g_{n m}(t) .
$$

The various contributions are modeled as follows.

1) Path loss and static shadowing: The path loss and static shadowing combined in $L_{n m}$ are modeled as outlined by (17) and by considering that obstruction loss $\bar{S}_{O}$ is a zero-mean Gaussian variable of standard deviation $\sigma_{\bar{S}_{o}}$, that $\bar{s}_{s}$ is SOSFdistributed for static nodes, and $\bar{S}_{s}=0$ for mobile scenarios.

2) Dynamic shadowing: $\tilde{S}_{n m}(t)$ is a time-varying zeromean Gaussian variable, whose standard deviation $\sigma_{\tilde{S}}$ is modeled differently for nomadic and mobile links (see Table III). The shadowing temporal auto-correlation is modeled as a decreasing exponential, whose decay time $\tau$ is listed in Table III and is also given alternatively by $d_{c} v^{-1}$ for mobile links, using the decorrelation distance value also listed in Table III and the node speed $v$. The shadowing correlation coefficient $C\left[n, m, n^{\prime}, m^{\prime}\right]$ is a function of the mobility scenario and the number of joint moving nodes, as detailed in Table III. Hence, for two links, joint shadowing time series are obtained 
TABLE III

MODEL PARAMETERS

\begin{tabular}{|c|c|c|c|c|c|c|}
\hline & $\mathrm{O} 2 \mathrm{I}$ & I2I (nomadic) & \multicolumn{2}{|c|}{ I2I (single mobile) } & \multicolumn{2}{|c|}{ I2I (double mobile) } \\
\hline$\eta$ & $2.27[25]$ & 1.75 & \multicolumn{4}{|c|}{$\overline{1.75}$} \\
\hline$\mu_{\bar{S}_{O}}[\mathrm{~dB}]$ & see $(26)$ & 0 & \multicolumn{4}{|c|}{0} \\
\hline$\sigma_{\bar{S}_{o}}[\mathrm{~dB}]$ & $8-10[22]$ & 4.6 & \multicolumn{4}{|c|}{4.6} \\
\hline$\left(\alpha_{s}, \beta_{s}\right)$ & $\operatorname{see}(18)$ & $\operatorname{see}(18)$ & \multicolumn{4}{|c|}{$=(0,1)$} \\
\hline$\sigma_{\tilde{S}}[\mathrm{~dB}]$ & see (14) & see (19) & \multicolumn{4}{|c|}{$=\sigma_{\bar{S}_{o}}$} \\
\hline$\tau[\mathrm{s}]$ & 1.74 & 2.5 & \multicolumn{4}{|c|}{$1.5($ speed of $0.3 \mathrm{~m} / \mathrm{s})$} \\
\hline$d_{c}[\mathrm{~m}]$ & non-applicable & non-applicable & \multicolumn{4}{|c|}{$0.45($ speed of $0.3 \mathrm{~m} / \mathrm{s})$} \\
\hline \multirow{2}{*}{$\left(\mu_{C}, \sigma_{C}\right)$} & \multirow{2}{*}{$(0,0.31)$} & \multirow{2}{*}{$(0,0.27)$} & common mov. & no (mov.) common & common mov. & no (mov.) common \\
\hline & & & $(0.28,0.47)$ & $(0.00,0.36)$ & $(0.29,0.39)$ & $(0.06,0.36)$ \\
\hline$K$ & see (15) & see $(20)$ & \multicolumn{4}{|c|}{ non-applicable } \\
\hline$(\alpha, \beta)$ & non-applicable & non-applicable & \multicolumn{2}{|c|}{ see $(23)$} & \multicolumn{2}{|c|}{ see $(22)$} \\
\hline
\end{tabular}

similarly to (30). Note that the correlation value for the I2Isingle mobile-common moving case in Table III cannot be found directly in Table II, as we have aggregated single mobile $\mathrm{Rx}$ and single mobile Tx cases to build Table III.

3) Fading: The small-scale fading $g_{n m}$ is best described in amplitude by a Ricean distribution in nomadic cases (the $\mathrm{K}$ factor being related to the distance, see (20)), while in mobile scenarios, the SOSF distribution is used to model the fading amplitude, with $(\alpha, \beta)$ randomly distributed as given in Table III. The SOSF random variable generator is obtained in a similar fashion as (31), based on its natural definition. Again, the fading processes between different links are modeled as independent variables.

\section{CONCLUSIONS}

This paper has presented a preliminary analysis and modeling of the outdoor-to-indoor and indoor-to-indoor channels based on experimental results at $2.4 \mathrm{GHz}$. The conclusive results can be summarized as follows.

- To accurately account for the different node mobility scenarios in distributed channels, we propose to model the channel based on a physically-motivated separation of static and dynamic shadowing. In nomadic channels, static shadowing further includes a spatial-fading term, which models the static multipath interference.

- The standard deviation of static obstruction shadowing (in all cases) and dynamic shadowing (in mobile cases) is about $4.5 \mathrm{~dB}$ indoors.

- In nomadic channels, the standard deviation of dynamic shadowing is positively correlated with the path loss.

- The dynamic shadowing correlation can be high (positively or negatively) and is related to the node mobility: the average correlation between two links is found to be more positive when both links share a common moving node. This is the first time such behavior is reported.

- For nomadic scenarios (both O2I and I2I), small-scale fading is well approximated by a Ricean or a mNakagami distribution, $K$ and $m$ decreasing with increasing distance/path loss.

- For I2I mobile transmissions, the temporal fading amplitude is modeled by a single distribution consisting of a weighted combination of Ricean and Double-Rayleigh distributions. We find that the Double-Rayleigh fading component is significantly stronger when both $\mathrm{Tx}$ and $\mathrm{Rx}$ nodes are moving as opposed to only one of them moving. This implies that the popular Rayleigh fading assumption, believed to be pessimistic, might in fact be too optimistic for the actual fading in cooperative doublemobile scenarios.

\section{REFERENCES}

[1] A. Sendonaris, E. Erkip, and B. Aazhang, "User cooperation diversity part I: System description,” IEEE Trans. Commun., vol. 51, no. 11, pp. 1927-1938, Nov. 2003.

[2] — - "User cooperation diversity - part II: Implementation aspects and performance analysis," IEEE Trans. Commun., vol. 51, no. 11, pp. 19391948, Nov. 2003.

[3] H. Ochiai, P. Mitran, H. Poor, and V. Tarokh, "Collaborative beamforming for distributed wireless ad hoc sensor networks," IEEE Trans. Signal Processing, vol. 53, no. 11, pp. 4110-4124, Nov. 2005.

[4] K. Nakao, T. Yamazato, M. Katayama, and H. Okada, "Cooperative transmission scheme in distributed sensor network for extension of transmission range," in Proc. 4th International Conference on Networked Sensing Systems, INSS '07, June 2007, pp. 89-92.

[5] E. Beres and R. Adve, "Selection cooperation in multi-source cooperative networks," IEEE Trans. Wireless Commun., vol. 7, no. 118-127, pp. 3013-3025, Jan. 2008.

[6] P. Agrawal and N. Patwari, "Correlated link shadow fading in multi-hop wireless networks," IEEE Trans. Wireless Commun., vol. 8, no. 8, pp. 4024-4036, Aug. 2009.

[7] R. Madan, N. Metha, A. Molisch, and J. Zhang, "Energy-efficient cooperative relaying over fading channels with simple relay selection," IEEE Trans. Wireless Commun., vol. 7, no. 8, pp. 3013-3025, Aug. 2008.

[8] P. Castiglione, S. Savazzi, M. Nicoli, and T. Zemen, "Impact of fading statistics on partner selection in indoor-to-outdoor cooperative networks," in Proc. ICC 2010 - IEEE Int. Conf. Commun., Cape Town, South Africa, May 2010.

[9] P. Soma, D. Baum, V. Erceg, R. Krishnamoorthy, and A. Paulraj, "Analysis and modeling of multiple-input multiple-output radio channels based on outdoor measurements conducted at $2.5 \mathrm{GHz}$ for fixed BWA applications," in Proc. ICC 2002 - IEEE Int. Conf. Commun., vol. 1, New York City, NY, May 2002, pp. 272-276.

[10] L. Ahumada, R. Feick, R. Valenzuela, and C. Morales, "Measurement and characterization of the temporal behavior of fixed wireless links," IEEE Trans. Veh. Technol., vol. 54, no. 6, pp. 1913-1922, Nov. 2005.

[11] J. Medbo, J.-E. Berg, and F. Harrysson, "Temporal radio channel variations with stationary terminal," in Proc. VTC 2004 Fall - IEEE Vehicular Technology Conf., vol. 1, 2004, pp. 91-95.

[12] P. Almers, K. Haneda, J. Koivunen, V.-M. Kolmonen, A. F. Molisch, A. Richter, J. Salmi, F. Tufvesson, and P. Vainikainen, "A dynamic multilink MIMO measurement system for $5.3 \mathrm{GHz}$," in Proc. 29th URSI General Assembly, Chicago, USA, Aug. 2008.

[13] F. Kaltenberger, M. Kountouris, D. Gesbert, and R. Knopp, "Correlation and capacity of measured multi-user MIMO channels," in Proc. IEEE Intl. Symposium on Personal, Indoor and Mobile Radio Communications (PIMRC), Cannes, France, Sept. 2008. 
[14] J. Karedal, A. Johansson, F. Tufvesson, and A. Molisch, "A measurement-based fading model for wireless personal area networks," IEEE Trans. Wireless Commun., vol. 7, no. 11, pp. 4575-4585, Nov. 2008.

[15] M. Gudmundson, "Correlation model for shadow fading in mobile radio systems," Electronics Letters, vol. 27, no. 23, pp. 2145-2146, 7 Nov. 1991.

[16] F. Graziosi and F. Santucci, "A general correlation model for shadow fading in mobile radio systems," IEEE Commun. Lett., vol. 6, no. 3, pp. 102-104, 2002.

[17] E. Perahia, D. C. Cox, and S. Ho, "Shadow fading cross correlation between basestations," in Proc. VTC 2001 Spring - IEEE Vehicular Technology Conf., vol. 1, 2001, pp. 313-317.

[18] J. Weitzen and T. J. Lowe, "Measurement of angular and distance correlation properties of log-normal shadowing at $1900 \mathrm{MHz}$ and its application to design of PCS systems," IEEE Trans. Veh. Technol., vol. 51, no. 2, pp. 265-273, 2002.

[19] N. Jalden, P. Zetterberg, B. Ottersten, H. Aihua, and R. Thoma, "Correlation properties of large scale fading based on indoor measurements," in Proc. IEEE Wireless Communications and Networking Conference, WCNC 2007, Mar. 2007, pp. 1894-1899.

[20] B. Bandemer, C. Oestges, N. Czink, and A. Paulraj, "Physically motivated fast-fading model for indoor peer-to-peer channels," Electronics Letters, vol. 45, no. 10, pp. 515-517, May 2009.

[21] N. Czink, B. Bandemer, G. Vazquez-Vilar, A. Paulraj, and L. Jalloul, "July 2008 radio measurement campaign: Measurement documentation," Tech. Rep., July 2008.

[22] E. Damosso and L. Correia, COST 231 - Digital mobile radio towards future generation systems. Brussels, Belgium: European Commission, 1999.

[23] R. Kattenbach, "Statistical modeling of small-scale fading in directional channels," IEEE J. Select. Areas Commun., vol. 20, no. 3, pp. 584-592, Apr. 2002.

[24] J. D. Parsons, The mobile radio propagation channel. London, UK: 2nd ed., Wiley, 2000.

[25] P. Kyosti, J. Meinila, L. Hentila, X. Zhao, T. Jamsa, C. Schneider, M. Narandzic, M. Milojevic, A. Hong, J. Ylitalo, V.-M. Holappa, M. Alatossava, R. Bultitude, Y. de Jong, and T. Rautiainen, "WINNER II Channel Models," European Commission, Deliverable IST-WINNER D1.1.2 ver 1.1, Sept. 2007. [Online]. Available: http://projects.celtic-initiative.org/winner+/WINNER2-Deliverables/

[26] "Propagation data and prediction models for the planning of indoor radiocommunication systems and local area networks in the frequency range $900 \mathrm{MHz}$ to $100 \mathrm{GHz}$," International Telecommunication Union, Recommendation ITU-R P.1238-4.

[27] S. M. Kay, Fundamentals of Statistical Signal Processing, Estimation Theory. Prentice Hall, 1993.

[28] C. Oestges and B. Clerckx, MIMO Wireless Communications. Elsevier Academic Press, 2007.

[29] J. Salo, H. M. El-Sallabi, and P. Vainikainen, "Statistical analysis of the multiple scattering radio channel," IEEE Trans. Antennas Propagat., vol. 54, no. 11, pp. 3114-3124, Nov. 2006.

[30] J. Cheng and N. C. Beaulieu, "Maximum-likelihood based estimation of the Nakagami m parameter," IEEE Commun. Lett., vol. 5, no. 3, pp. 101-103, Mar. 2001.

[31] C. Oestges and A. Paulraj, "Propagation into buildings for broadband wireless access," IEEE Trans. Veh. Technol., vol. 53, no. 2, pp. 521-526, Mar. 2004.

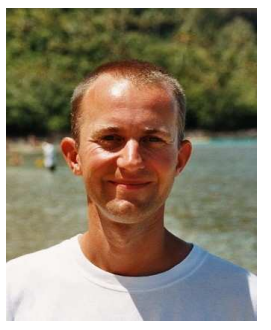

Claude Oestges (M'05) received the M.Sc. and $\mathrm{Ph} . \mathrm{D}$. degrees in Electrical Engineering from the Université catholique de Louvain (UCL), Louvainla-Neuve, Belgium, respectively in 1996 and 2000. In January 2001, he joined as a post-doctoral scholar the Smart Antennas Research Group, Stanford University, CA. From January 2002 to September 2005, he was associated with the Microwave Laboratory UCL as a post-doctoral fellow of the Belgian Fonds de la Recherche Scientifique (FRS). Claude Oestges is presently a FRS Research Associate and Assistant

Professor at UCL. He is the author or co-author of one book and more than 120 research papers and communications, and was the recipient of the 1999-2000 IET Marconi Premium Award and of the 2004 IEEE Vehicular Technology Society Neal Shepherd Award.

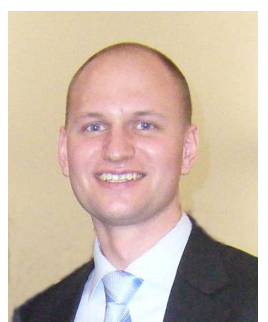

Nicolai Czink received his Dipl.-Ing. (M.S.) degree in 2004 and Dr.techn. (Ph.D.) degree in 2007, both from Vienna University of Technology, Austria, with distinction. His Ph.D. thesis received an award from the Austrian Electrotechnical Association (OVE). After his Ph.D., he joined Stanford University as a Postdoctoral Researcher on an Erwin Schrödinger Fellowship of the FWF Austrian Science Fund. He is currently Senior Researcher at the FTW Telecommunications Research Center Vienna, Austria, working on channel modeling, cooperative communications, and intelligent transportation systems. Nicolai Czink is the author of more than 50 research papers and communications in international journals and conferences.

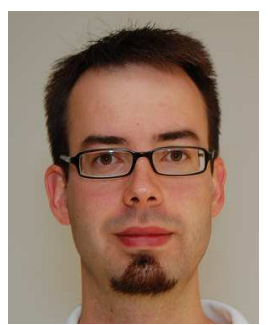

Bernd Bandemer (S'06) received his Dipl.-Ing. (MS) degree in Electrical and Computer Engineering in 2006 from Ilmenau University of Technology, Ilmenau, Germany. In 2003/04, he was awarded a Fulbright scholarship to study at Purdue University, West Lafayette, Indiana. His experience outside academia includes working as a research intern at Intel Corporation (Santa Clara, California), at Nokia Research Center (Helsinki, Finland), and at the German Aerospace Center (Munich, Germany). He is currently pursuing a Ph.D. degree in Electrical Engineering at Stanford University. His research is centered around interference and cooperation in wireless networks, from both the information-theoretic and signal processing viewpoints.

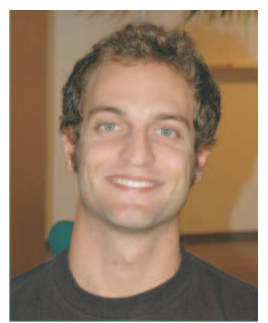

Paolo Castiglione received the M.Sc. degree (with honors) in Information Technology in 2008 from Dipartimento di Elettronica e Informazione, Politecnico di Milano, Italy. In 2006, he was an exchange student at the Munich University of Technology, Germany, where he also joined Siemens speech processing group. He is presently a Ph.D. student at Vienna University of Technology and researcher at the FTW Telecommunications Research Center Vienna, Austria. His current research interests include signal processing aspects for digital wireless communications, with a major focus on cooperative networking.

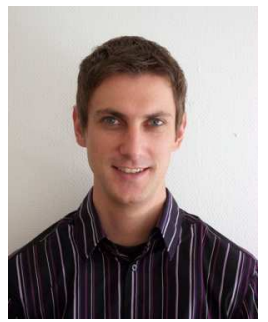

Florian Kaltenberger (S'05-M'08) received his Dipl.-Ing. degree and his Ph.D. degree both in technical mathematics from the Vienna University of Technology in 2002 and 2007, respectively. In 2002, he started as a Research Assistant at the Vienna University of Technology, Institute for Advanced Scientific Computing, working on distributed signal processing algorithms. In 2003, he joined the wireless communications group at the Austrian Research Centers GmbH. Since 2007, he is Research Engineer at Eurecom, Sophia-Antipolis, France where he is working on the Eurecom real-time open-source experimental platform OpenAirInterface.org. His research interests include signal processing for wireless communications, MIMO communication systems, receiver design and implementation, MIMO channel modeling and simulation, and hardware implementation issues.

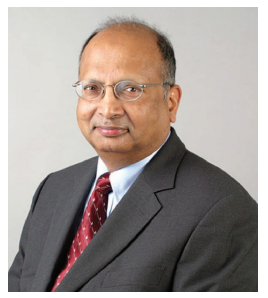

Arogyaswami Paulraj (F' 91) received the Ph.D. degree from the Naval Engineering College and the Indian Institute of Technology, Delhi, India, in 1973. He has been a Professor with the Department of Electrical Engineering, Stanford University, CA, sine 1993, where he supervises the Smart Antennas Research Group. In 2004, he co-founded Beceem Communications, Inc. to develop base band and RF chips sets for WIMAX 802.16e standard. His research has spanned several disciplines, emphasizing estimation theory, sensor signal processing, parallel computer architectures/algorithms, and space-time wireless communications. His engineering experience includes development of sonar systems, massively parallel computers, and, more recently, broadband wireless systems. He is the author of over 400 research papers and holds 41 patents as well as several awards. He is a Member of the US National Academy of Engineering, the Royal Swedish Academy of Engineering Sciences, the Academy of Sciences for the Developing World and the Indian Academy of Engineering 